\title{
Conductivity Effect on the Capacitance Measurement of a Parallel-Plate Capacitive Sensor System
}

\author{
Peyman Azimi Anaraki \\ Department of Physics \\ Islamic Azad University, Takestan-Iran \\ e-mail:peyphysics2004@yahoo.co.uk
}

Received 31 January 2011, Revised 2 June 2011, Accepted 23 August 2011

\begin{abstract}
bstractA
In this article design and operation of a parallel-plate capacitive sensor based on the dielectric capacitance and conductance change of the gap medium is reported. The designed system was used to determine characteristics of different water liquids as a result of the capacitance variations. The air gap capacitance is measured and then by filling the gap with a liquid the capacitance is monitored for different liquids. Proposed sensor is used for the distilled, tap, boiled, and salt water measurements and the capacitance results are compared. A big difference about $45.5 \mu \mathrm{F}$ in capacitance values for the salt and distilled water shows a high sensitivity, which can be used to recognize different water liquids. The experimental results are promising for water liquids and verify the successful operation of such a device as a liquid sensor, a useful method for checking the electrical quality of the water that is required for different applications.
\end{abstract}

Keywords: Sensor; Capacitance; Permittivity change; Conductivity; Water liquids.

\section{Introduction}

There has been a great deal of interest in the development of precise capacitive sensor in recent years. Different reports on the design, characterization, operation and possible applications of such devices have been given by the author ${ }^{1,2)}$ and others $^{3-7)}$. A CMOS-compatible capacitive high temperature pressure sensor was reported ${ }^{8)}$. Measurements in the range of $50-340{ }^{\circ} \mathrm{C}$ for temperature and pressure for the range of $0-125$ bar were performed by such a small sensor. Also for pressure measurement in harsh environment a capacitive differential sensor has been reported, which operated for the pressure range of 0-1 bar ${ }^{9)}$. In another study, a monolithically integrated surface micromachined touch mode capacitive pressure sensor was reported $^{10)}$. Their fabrication was reported to be a fully CMOS-compatible touch mode capacitive pressure sensor. The frequency and voltage out put of such sensors were about 5-25 Hz/psi, and 10-50 $\mathrm{mV} / \mathrm{psi}$ in the linear pressure range of 8-60 psi.

Capacitive sensors have been used in many industrial applications to control processes and in machine diagnostic tasks. However several problems including stray capacitance, baseline drift, stability and sensitivity have motivated the development of new transducers and measuring systems. To alleviate some of the problems in this field a variety of the capacitive sensor systems have been developed and reported. In this respect, for example, the effects of a guard ring electrode on the operation of a capacitive transducer have been investigated ${ }^{11)}$. Development of a three-dimensional capacitance imaging system for measuring the density of fluidized beds was reported $^{12)}$. In another report, design and operation of a capacitive sensor for water content monitoring in a production line was presented ${ }^{13)}$. Design and performance of simple capacitive sensors for mass measurement is given by Ref. ${ }^{3)}$. Among several industries, the scale industry has been developing rapidly and there are demands for weighting devices with good qualities at low prices.

In another report, capacitance sensors have been used for the measurement of the phase volume fraction in two-phase pipelines ${ }^{14)}$. The effect of phase distribution or flow pattern was considered for determination of the volume fraction in two phase pipe-line by using the capacitance measurements. They have shown that the capacitance measured depends not only on the volume fraction but also on the phase distribution and they have shown such effect by an example. In that article they described the resulting capacitance when the electrodes are half filled vertically or horizontally similar to the series or the parallel capacitive forms.

On the other hand many researchers have focused on the development of the readout circuits. The goal of such research has been to introduce a readout circuit that can be used for low-noise operation with the cancellation of the operational amplifier1/f-noise and offset voltage ${ }^{15)}$. A new capacitive-to-phase conversion technique for measuring very small capacitance changes has been reported $^{16)}$. This method provided a powerful mean for recording very small capacitance changes. Much progress has been made over the last years in developing the capacitor transducers and complementing measuring circuits. For the precision in instrumentation and measurements, the small capacitances to be measured are in the range of 0.01$10 \mathrm{pF}$ with a required resolution of better than 0.01-10 $\mathrm{fF}$. This requirement along with other considerations 
such as environmental effects, structural stability, and standardization challenges the development of a much more sensitive and reliable capacitance sensor systems $^{17)}$.

\section{Experiment}

The experimental setup, measurement method, materials and sample preparations are described in this section.

\subsection{Setup}

Capacitance measurement system in general includes a sensing probe and a measuring module. Our experimental setup is a simple one, which uses the capacitive sensing probe and the measuring module as shown in Figure 1. The experimental arrangement includes the parallel-plate capacitive sensor, two digital multimeter (DMM) modules (SANWA, PC 5000), and a PC. As shown in Figure 1, one of the digital multimeters is used for the capacitance measurement and a similar one together with a temperature probe (T-300PC) is used for the temperature measurements ${ }^{18)}$. The software (PC Link plus) allows one to log measuring data into $\mathrm{PC}$ through RS232 port with digital multimeter PC series. The operation of this software is possible by using any operational system such as the windows 98, NT4.0/2000/ME/XP versions. It provides function for capacitance measurements using the charge/discharge method and capacitance in the range of $0.01 \mathrm{nF}$ to $9.99 \mathrm{mF}$ can be measured with a resolution of about $0.01 \mathrm{nF}$. The nominal input impedance of the DMM is about $10 \mathrm{M} \Omega$ and $30 \mathrm{pF}$. The specified accuracy of the DMM for 50.00-500.0 $\mathrm{nF}$ capacitance range is about \pm $(0.8 \% \mathrm{rdg}+3 \mathrm{dgt})$ and $\pm(2 \% \mathrm{rdg}+3 \mathrm{dgt})$ for the 50.00 $\mu \mathrm{F}$ range. The temperature probe consists of a platonic thin thermoresistor $\left(1000 \Omega\right.$ at $\left.0{ }^{\circ} \mathrm{C}\right)$ with a temperature measurement range of -50 to $300{ }^{\circ} \mathrm{C}$. The response time of this probe is about 7 seconds and offers an accuracy of about $\pm 1.9{ }^{\circ} \mathrm{C}$ in temperature recording.

The proposed capacitive probe shown in Figure 1 consists of two parallel-plate that is separated by $2 \mathrm{~mm}$, the total length of electroplates, $18.5 \mathrm{~cm}$, the width of the electroplates is $17.5 \mathrm{~cm}$. when the space between the capacitor plates is completely filled with the water liquids, the capacitance is:

$$
C=\varepsilon \frac{A}{d}
$$

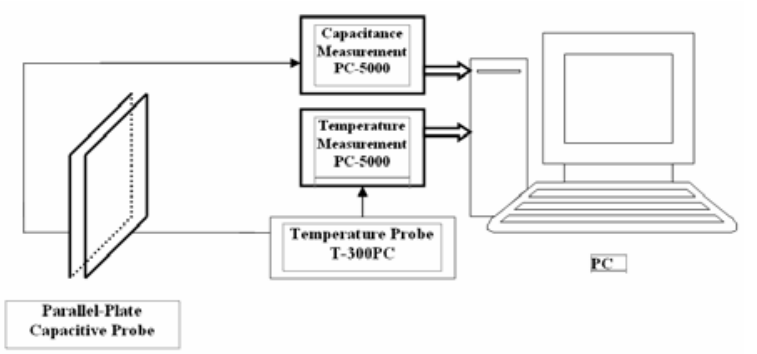

Figure 1. Block diagram of the experimental arrangement for capacitive sensor.
Where $\varepsilon$ is permittivity of the dielectric medium between the plates. A is electrode area and $d$ is gap distance between the electrodes. However Eq. (1) is only valid when $A>>d$. several problems, such as edge effect, can cause deviation in the actual capacity from the one obtained in Eq. (1). For this reason, various attempts have been made to design different transducers in order to reduce these effects.

\subsection{Measurement method}

Depending on the capacitance electrode configuration of the sensor the equivalent circuit can be considered for the case of invasive (direct contact between the metal electrode and liquid), and noninvasive (no contact between the metal electrode and liquid) sensors. In a simple form if we consider a uniform liquid with the given permittivity and conductivity, the equivalent circuits for the case of non-invasive and invasive sensors can be considered $^{19)}$.

It must be mentioned that the given capacitance value is the measured value by the charge transfer reading circuit and fluid capacitance must be deduced from the measured values. Also noted that the capacitance sensing is affected by the conductivity variations of the components ${ }^{20)}$. This conductivity problem has been the main concern in the field of dielectric measurements and several attempts have been made to compensate for such variation and for a simple case the effect of conductivity is presented by a resistive element in parallel with the sensor capacitance. However, for sensors using non-invasive electrodes and those measuring two-component fluids; sensor system must be represented by more complex equivalent circuit models. As a result an investigation into the effects of component conductivity should be done for precise measurements.

Equivalent circuits for the invasive and noninvasive cases are shown in Figure 2(a) and Figure 2(b), respectively. In the invasive situation when there is a contact between the metal and liquid it is equivalent to a circuit consisting of a capacitor $C_{x}$ in parallel with a resistor $R_{x}$. In this analysis $R_{x}$ representing the resistance of the fluid due to its conductivity effect, and $\mathrm{C}_{\mathrm{x}}$ shows its capacitance as a result of its permittivity. For the non-invasive case as shown in Figure 2 (b) an extra capacitor $\mathrm{C}$ is considered in series with $\mathrm{R}_{\mathrm{x}}$ and $\mathrm{C}_{\mathrm{x}}$, which are acting in parallel. As can be seen, measured capacitance element is depending on both $C_{x}$ and $R_{x}$ of the fluid under measurement.

In general a variety of techniques have been employed for measuring the absolute and relative capacitance changes. Oscillation, Resonance, charge/discharge, AC Bridge, and capacitive-to-phase conversion are the most common methods for such capacitance measurements. Since the measurement module uses the charge/discharge (C/DC) circuit, therefore, this method is described here. The charge/discharge operation is based on the charging of 
an unknown capacitance under study $\mathrm{C}_{\mathrm{x}}$ to a voltage $V_{c}$ via a CMOS switch with resistance $R_{\text {on }}$ (Figure 3 a) and then discharging this capacitor into a charge detector via a second switch (Fig3 b).

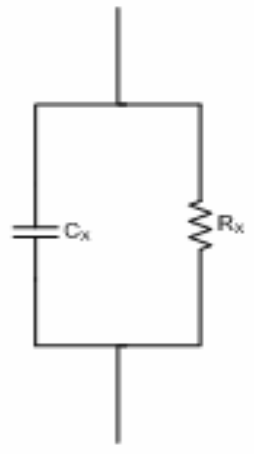

(a)

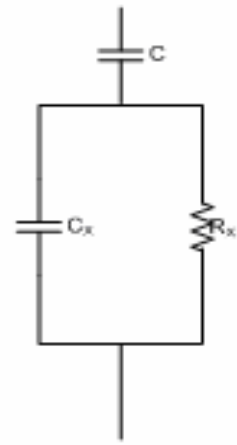

(b)
Figure 2. Equivalent circuits for the invasive (a) and non-invasive (b) electrode arrangements.

A DMM with the given specification based on the charge/discharge operation is used here for the capacitance measurements. This capacitance measuring module is capable of measuring precisely the capacitance values in the range of $0.01 \mathrm{nF}$ to 50 $\mathrm{mF}$.

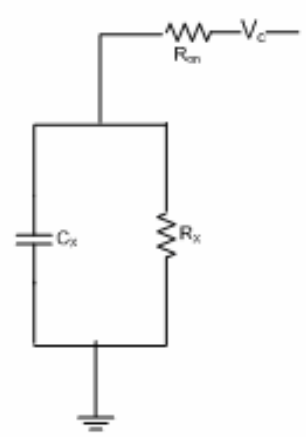

(a)

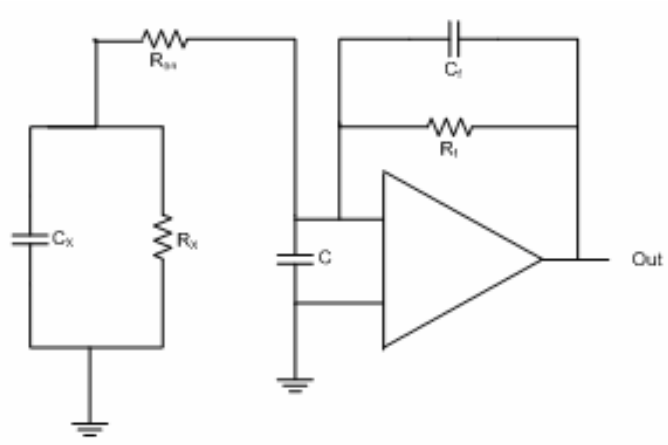

(b)

Figure 3. Schematics of the capacitance charge (a) and discharge (b) processes.

The capacitance measurements for the parallelplate capacitive probe shown in Figure 1 depend on the permittivity, $\varepsilon$, of the liquid and its resistance factor that depends only on the conductivity, $\sigma$, of the liquid. Thus one can write:

$$
\begin{aligned}
& C_{x}=f_{1}(\varepsilon), \\
& R_{x}=f_{2}(\sigma) .
\end{aligned}
$$

The capacitive element $\mathrm{C}$ is obtained only by the insulation of the electrodes and reducing the conductivity effect. As described, in general there are invasive and non-invasive electrode arrangements. For the case of non-invasive sensors, in measuring capacitance of a liquid, the effect of resistive component is usually very small because of the dielectric insulator. For the invasive sensors the effect of $R_{x}$ on the measurement of $C_{x}$ can not be neglected and the effect of conductivity of the liquid must be considered in analysis. However, the effect of $R_{x}$ can be negligible if the on resistance of the charge switch $\mathrm{R}_{\text {on }}$ is small compared with $\mathrm{R}_{\mathrm{x}}$, and if the discharge time, which is determined by the switching-on time of the resistance of the discharging switch, is short compared with the time constant given by $\mathrm{R}_{\mathrm{x}} \mathrm{C}_{\mathrm{x}}$.

To analyze electrical conduction of the tested water liquids, additional measurements were made on the electrical conductivity (EC) and total dissolved solid (TDS) density of the samples used in this experiment. In conductivity meters usually measurements is made by placing a cell (probe) in an electrolytic solution. The cell consists of two electrodes of specific size, separated at a specific distance that defines the cell $\mathrm{K}$ factor. The conductivity of a liquid is determined from the ratio of current to the voltage between the two electrodes.

For measurements a conductivity meter (Hach Company, sension5) is used ${ }^{21)}$, which is useful for a variety of applications such as water quality, measuring salinity (a measure of dissolved salts in a given mass of solution), acids, bases, and other qualities of aqueous samples. The meter features a digital LCD display that simultaneously displays temperature and other measurement results. The specifications of this meter are as follows: Conductivity range of $0-199.9 \mathrm{mS} / \mathrm{cm}$ and resolution of $0.01 \mu \mathrm{S} / \mathrm{cm}$ to $0.1 \mathrm{mS} / \mathrm{cm}$, respectively, for the selected measurement ranges. The ranges for TDS are: $0-50 \mathrm{~g} / \mathrm{L}$ with a resolution of $0.1 \mathrm{mg} / \mathrm{L}$ to $0.1 \mathrm{~g} / \mathrm{L}$ for different ranges. Measurement range for salinity is 0 $42 \mathrm{ppt}$ with a resolution of $\pm 0.1 \mathrm{ppt}$ and an accuracy of $\pm 0.1 \mathrm{ppt}$. The measuring temperature range is from $-10{ }^{\circ} \mathrm{C}$ to $105{ }^{\circ} \mathrm{C}$. The accuracy for conductivity is \pm $0.5 \%$ of range, for TDS is $\pm 0.5 \%$ of full scale, and for temperature is $\pm 0.3{ }^{\circ} \mathrm{C}$ for $0-70{ }^{\circ} \mathrm{C}$ and $\pm 1.0{ }^{\circ} \mathrm{C}$ for $70-105{ }^{\circ} \mathrm{C}$. Calibration of this device is based on a standard $\mathrm{NaCl}$ solution having a conductivity of 1000 $\mu \mathrm{S} / \mathrm{cm}$ at $25^{\circ} \mathrm{C}$.

\subsection{Samples and procedures}

Fortunately this experiment does not require many reagents and complicated chemical procedures for sample preparation. In this experiment one type of samples was used including the single aqueous solutions. The single phase solution includes the distilled water, tap, boiled, and mineral drinking water samples. The distilled water used in this experiment 
was made by an apparatus operating based on the boiling technique. All other agents and liquids were at regular grade purchased from the related suppliers. For the water salt preparation regular grade salt $(\mathrm{NaCl})$ was used for the preparation of a sample with the concentration of about $\mathrm{mg} / \mathrm{L}$.

\section{Results}

The minimum reliable limit of DMM is about $0.01 \mathrm{nF}$, therefore the direct results for air reading were not so reliable. However, when the gap is filled with a liquid, since the dielectric constant of the fluid is larger than that of the air, then the measured capacitance value is increased and the capacitance value is acceptable for these water liquids. As described, our measuring apparatus is operating under the charge/discharge technique. In order to test the precision of the capacitance measuring module, in the first experiment the capacitance of air is measured $(0.12 \mathrm{nF})$.

The air gap capacitance of the probe is around $0.09-0.12 \mathrm{nF}$. The air gap capacitance for the designed probe is about $0.11 \mathrm{nF}$. However, as described this value is different from the calculated value from Eq. (1).It must be pointed that the theoretical value is for the cell that air gap between two plates is very small (maximum $2 \mathrm{~mm}$ ) and the mentioned difference seems reasonable. In the next test the capacitance value for the distilled water is measured. In the first case the measured value is about $6.5 \mu \mathrm{F}$. The theoretical capacitance due to a dielectric constant of about 80 for the distilled water leads to a dielectric capacitance value of $0.348 \mathrm{nF}$, which is much less than the measured value. This leads to the fact that the measured value is not the capacitance value of the distilled water and must be compensated for the conductance effect.

In the next study to see the capacitance variation of the different water liquids a series of measurements are performed and results are shown in Figure 4. As can be seen in Figure 4, the distilled water shows the lowest measured value and the water salt solution shows the highest $(61.8 \mu \mathrm{F})$ value among the tested liquids.

For the case of distilled water the capacitance value starts from the $6.5 \mu \mathrm{F}$ and reaches to about 15.3 $\mu \mathrm{F}$ in a few seconds time interval. As described conductivity of the liquid material has important role in the capacitance measurements and as a result in sensor operation. From Figure 4 two major points can be concluded. First, the measured capacitance value recorded by the module is not only the liquid capacitance but also the capacitance due to the liquid conductance. Thus the present probe provides a sensitive cell for the investigation of the conductance effect in such measurements. Among the tested water liquids the salt water shows the highest measured capacitance value of $61.8 \mu \mathrm{F}$ while the distilled water shows the lowest measured value of $15.3 \mu \mathrm{F}$.
Considering a notable difference $(45.5 \mu \mathrm{F})$ for salt water in capacitance values for the different water liquids is indicative of the high sensitivity of the reported sensor.

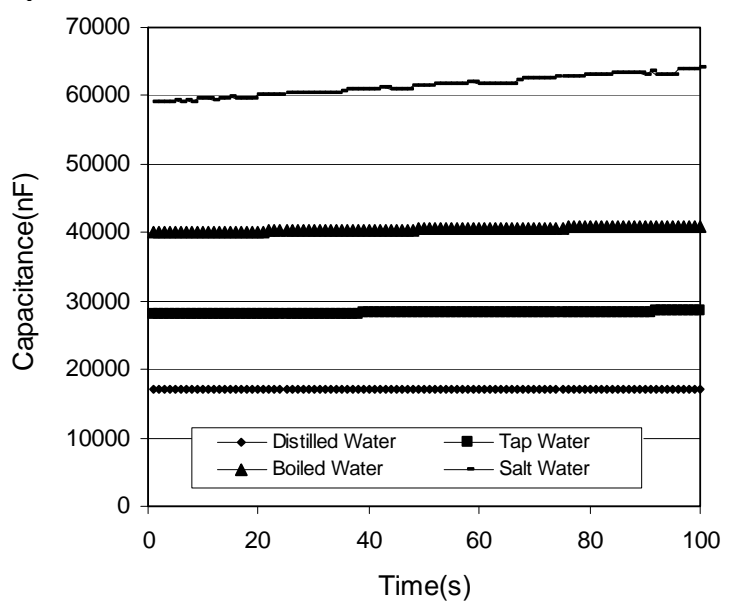

Figure 4. Capacitance values for different water liquids.

Second point is that the measured values are relatively constant for the distilled, boiled, and tap water measurements, but larger fluctuations are observed in the salt water measurements. This leads to the fact that such fluctuations are certainly due to the conductivity effect. The designed sensor, therefore, can be used to investigate dynamic behavior of the liquid in such measurements. It can be concluded that the measured capacitance values are due to the dielectric term and a second term related to the conductivity of the liquid. For the distilled water the conductance effect is minimum while for the salt water has the considerable value. For the air gap such term vanishes and the measured capacitance is only due to the first $f_{1}$ term as shown in Eq. (2). Thus the fluid capacitance can be obtained from the difference of the measured capacitance values and the $f_{2}$ term, Eq. (3), due to the conductivity effect.

A comparison of the results for different water liquids at given temperature is listed in Table 1. The electrical conductivity (EC), total dissolved solid (TDS), capacitance(C), and temperature (T) are listed in Table 1. As can be seen in Table1 for the water samples, the EC factor is increased as well as the TDS in the given order for the tested water liquids. Considering the capacitance value with the EC values confirms our argument about the effect of the electrical conductance on the capacitance measurements. It is noted that there is a relation between the increase of the electrical conductivity of the liquids and the increase of the measured capacitance. Looking at Table 1 , it is noted that the salt water posses the highest EC value of $22100 \mu \mathrm{S} / \mathrm{cm}$ while the distilled water shows the least EC value of $9.2 \mu \mathrm{S} / \mathrm{cm}$ at the same temperature. 
Table 1. Comparison of the capacitance values for different water liquids.

\begin{tabular}{|c|c|c|c|c|}
\hline Sample & $\begin{array}{c}\text { Electrical } \\
\text { Conductivity } \\
\mathbf{( \mu S / m )}\end{array}$ & $\begin{array}{c}\text { Total Dissolved Solid } \\
\text { Density } \\
(\mathbf{m g} / \mathbf{L})\end{array}$ & $\begin{array}{c}\text { Measured } \\
\text { Capacitance } \\
(\boldsymbol{\mu F} \mathbf{)}\end{array}$ & $\begin{array}{c}\text { Temperature } \\
\left({ }^{\circ} \mathbf{C}\right)\end{array}$ \\
\hline Distilled Water & 9.2 & 11.3 & 19.6 & 25.4 \\
\hline Boiled Water & 590 & 293.2 & 39.5 & 25.6 \\
\hline Mineral Water & 443 & 220.5 & 36.1 & 25.6 \\
\hline Tap Water & 375 & 190.1 & 29.2 & 25.1 \\
\hline Salt Water & 22100 & 13100 & 61.4 & 25.4 \\
\hline
\end{tabular}

* For the stabilized condition

In Figure 5 the repeatability of the results for the reported capacitive cell sensor is shown. Such parameter indicates the ability of the sensor to reproduce output reading when operating under the same ambient condition. To provide such a condition a number of 100 measurements were made consequently for a series of readings. Such measurements are performed for all the samples and for the case of air-gap are shown in Figure 5. The reproducibility of the measured values for such consequent measurements is estimated to be about $1 \%$ of the full scale value.

Stability of a sensor is another important parameter, which is described in this study. In general such factor shows the ability of the sensor to maintain its performance characteristics for a certain period of time. In this experiment the capacitance values for the air-gap and liquid-gap cases are measured for a period of $100 \mathrm{~s}$ in $1 \mathrm{~s}$ increment. Both measured values (dry and wet cases) show a good stability for this period of time, which is about $1 \%$ of the full scale. The repeatability of the reported sensor is also investigated. Such parameter indicates the ability of the sensor to reproduce output reading when operating under the same condition. To provide such a similar ambient conditions a number of about 100 measurements were made consequently. The error of the measured values for the consequent measurements is estimated to be about $2 \%$ of the full scale. Air-Reproducibility

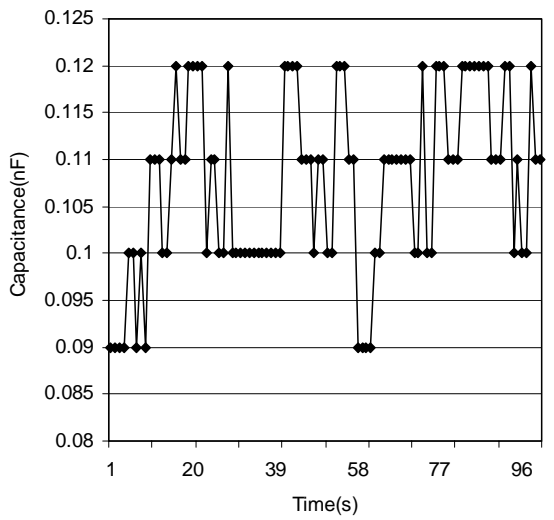

Figure 5. Reproducibility of the result for the sensor.
To understand the importance of permittivity variation as a result of different liquids, in Figure 6 we consider the measured values for a period of $100 \mathrm{~s}$ in $1 \mathrm{~s}$ increments. As indicated in Figure 6 the average permittivity values given for different liquids are $1246.455,868.625,721.654,421.325 \frac{n F}{m}$, for salt, boiled, tap, and distilled water. Generally the permittivity is more dependent on temperature and purity of materials. As described the highest measured permittivity value is $1246.455 \frac{\mathrm{nF}}{\mathrm{m}}$ for salt water while the lowest measured permittivity is 421.325 for distilled water, considering a significant difference $\left(825.13 \frac{\mathrm{nF}}{\mathrm{m}}\right)$ in permittivity value for different liquids is indicative of the highest sensitivity of the reported sensor, then finally from Figure 6 we find out stability and sensitivity are two major properties for the application of this sensor.

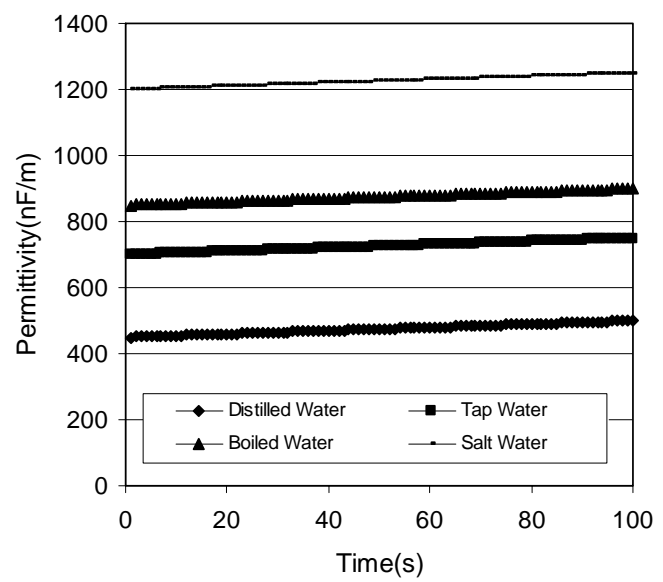

Figure 6. Comparison of the permittivity values for different water liquids as a function of time.

To see the capacitance variation as a result of different water liquids a series of measurements are performed and the measured values are shown in Figure 4. As can be seen in Figure 4, results of recorded capacitance values for distilled, boiled, tap, and salt waters as a function of measuring time are presented. The average capacitance values are 
$19278.54 \mathrm{nF}, 29345.26,41235.47,61516.12 \mathrm{nF}$ for distilled, tap, boiled, and salt waters, respectively. As described before, these values are just the measured capacitance values, which are much higher than the capacitance value of the liquids. The capacitance value of the distilled water is around $3 \mathrm{pF}$ as reported in Ref. ${ }^{20)}$. It is noted that the measured values are mainly related to the conductance term and can be used effectively to monitor the conductance and its dynamic developments in liquid filling.

For the case of distilled water the capacitance value starts from the $6.5 \mu \mathrm{F}$ and reaches to about $19.59 \mu \mathrm{F}$ in a $450 \mathrm{~s}$ time interval (as can be seen in Fig7.). As indicated the value given for the distilled water in Figure 4 is for the case of the stabilized final condition. However, for the other water liquids the stable condition is reached quickly in about a few seconds and then measured values are plotted. As described conductivity of the liquid material has important role in the capacitance measurements and as a result in sensor operation.

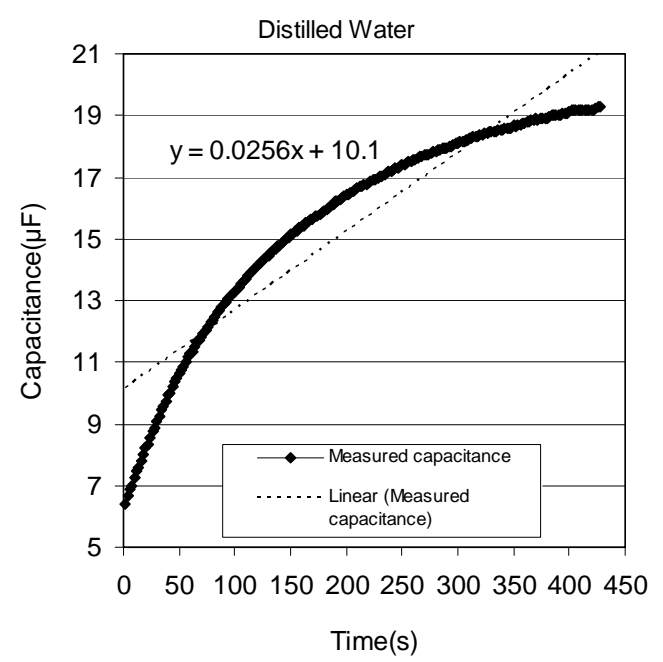

Figure 7. Capacitance measurement as a function of time for distilled water.

From Figure 4 two major points can be concluded. First, the measured capacitance signal value recorded by the module is not only the liquid capacitance but also the output due to the liquid conductance. Such measured value is due to both of the dielectric reactance capacitance, conductance term, and the stray terms. Thus the present device provides a sensitive probe for the investigation of the conductance effect in such measurements. Among the tested water liquids the salt water shows the highest measured capacitance value of $61.51 \mu \mathrm{F}$ while the distilled water shows the lowest measured value of $19.52 \mu \mathrm{F}$. Considering a notable difference (41.99 $\mu \mathrm{F}$ for salt water) in capacitance values for the different water liquids is indicative of the high sensitivity of the reported sensor. To verify this point in another experiment the capacitance and the resistance of the sensor cell filled with different liquids are measured at the recorded temperature and the results of these measurements are listed in Table1. As can be seen the reciprocal of the resistance shown as conductance is also obtained and shown in Table1. It is noted that there is a direct relation between the increase of the conductance and that of the measured capacitance output.

Second point is that the measured values are relatively constant for the distilled, boiled, and tap water measurements, but as indicated in Figure 8, larger fluctuations are observed in the salt water measurements. This leads to the fact that such fluctuations are certainly due to the conductivity effect, which are more pronounced for the salt water due to higher conductivity. As can be seen in Figure 4 , such effect is smaller for the more pure distilled water, but the presence of conductive impurity plays a more important role in the case of salt water. The designed sensor, therefore, can be used to investigate dynamic behavior of the liquid in such measurements. Salt Water

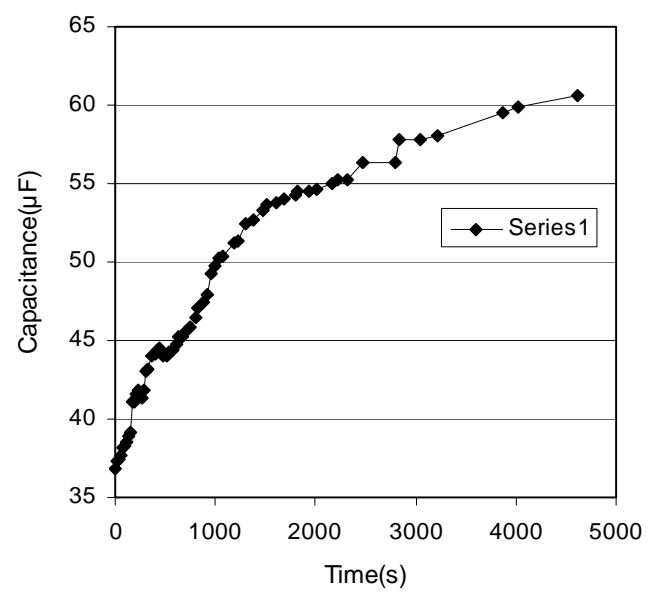

Figure 8. Variation of the capacitance as a function of time for salt water.

The variation of the capacitance as a function of time for distilled water is presented in Figure 7. The capacitance value starts from $6.5 \mu \mathrm{F}$ at about $5 \mathrm{~s}$ and reaches to about $19.27 \mu \mathrm{F}$ at $450 \mathrm{~s}$. Using the add trend line option of the program a fitted curve (dashed-line ) is also presented in Figure 7. As can be seen in Figure 7, the measured value shows a rapid growth and reaches a stable condition in about 7.5 minutes. Comparing the experimental results with the fitted polynomial curve it is noted that there is a good agreement between the measured values and the fitted one for the rising part and only there is a little deviation for the later times. This time variation situation tested several times and it was reproducible for the distilled water. Such dynamic behavior can be explained as following. The physical model of the situation when there is no contact between the electrode metal and liquid is equivalent to a circuit consisting of a capacitor of reactance $X_{1}$ in series with a resistor $R_{x}$ (liquid resistance). When there is a contact between the electrode metal and liquid the equivalent circuit is different from the first case and 
can be considered to be a capacitor of reactance $X_{2}$ in parallel with a resistor $R_{b}$ both in series with a resistance of $R_{2}$, in which $R_{b}$ is the resistance of the liquid bridge and $R_{2}$ is the resistance of the main bulk of the liquid. At the start of the liquid filling the bridge resistance is not fully formed and the measured value is low. As time goes on the bridge resistance is build up and the reading value is rapidly increased. After some time the contact resistance is fully formed and the output reading of the sensor is almost constant. The reported sensor also provides a mean to investigate the dynamic behavior of such liquid bride resistance formation. Comparing our results for the distilled, tap, and salt water it was noticed that such a bridge contact is formed very quickly for the samples with high conductivity effect, which verifies the described bridge contact physical model.

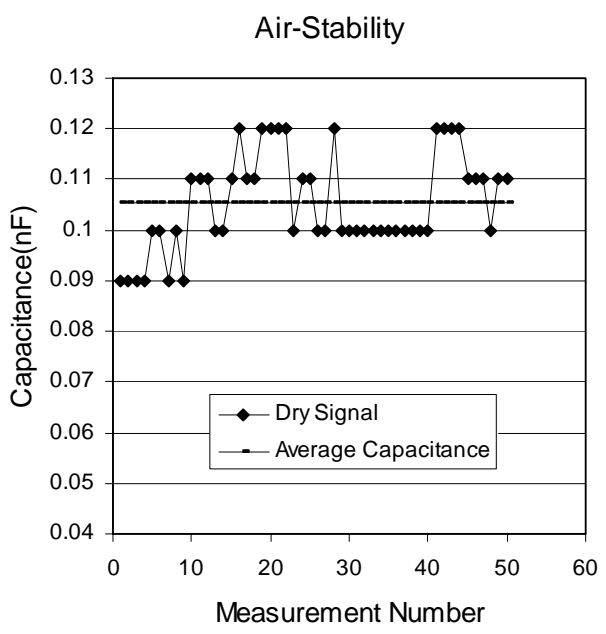

Figure 9. Reproducibility of the results for the designed sensor.

Precision is defined as a measure of the reproducibility of the measurements that is considered as a figure of merit for such a sensing device. Figure 9 shows the repeatability of the reported sensor. In this sensor such a parameter indicates the ability of the capacitive sensor to reproduce output reading when the same measurement is applied to it consequently, under the same condition. To provide such a similar ambient conditions measurements were made consequently for a series of 50 readings. The output capacitances for such repeated measurements are shown in Figure 9, which varies from $0.09 \mathrm{nF}$ to 0.12 $\mathrm{nF}$ at most. The average signal value calculated to be 0.1054 with the standard deviation of 0.009521 in measurements. For a better comparison average value of capacitance measurement (Dry signal) is also indicated as a dashed line in Figure 9.

\section{Conclusion}

As described, the invasive sensor such as the one reported here provided a useful mean to study the conductance effect of the reactance capacitance and its role in capacitance measurements. On the other hand, the non-invasive design is more suitable for studies concerning the permittivity effect of the liquids. In either case one needs to minimize the impact of the other factor in order to obtain more reliable readings. Considering the results both of the capacitive probe and the capacitance readout circuits have important role in the accuracy of the absolute measured values for different type of conducting and non-conducting liquids.

For the case of a gap material with low conductivity the charge/discharge method was used with the proposed parallel-plate probe to measure capacitance. This was a useful method for the checking the quality of the water, which is required for different applications. Although the results reported here were for the water liquids, but the reported sensor could be effectively implemented for the study of other conducting liquids such as industrial oils, and liquids, which have wide applications as lubricator, electric insulator, and cooling agents with a notable conductance contribution. Arrangement described also can be used for liquid mixture checking and also to see effect of impurities in the water solution.

\section{Acknowledgements}

This study was supported by the Islamic Azad University Branch of Shoushtar and Takestan. The authors like to acknowledge this support and thank the office of Vice President for Research and Technology.

\section{References}

1. H. Golnabi and P. Azimi, Simultaneous measurements of the resistance and capacitance using a cylindrical sensor system, Mod. Phys. Lett B, 22:8, 595, 2008.

2. H. Golnabi and P. Azimi, Design and performance of a cylindrical capacitive sensor to monitor the electrical properties, J. Applied Sci., 8:9, 1699, 2008.

3. H. Golnabi, Simple capacitive sensors for mass measurements, Rev. Sci. Instrum, 68, 1613, 1997.

4. O. Akar, T. Akin and K. Najafi, A wireless batched sealed absolute capacitive pressure sensor, Sens. Actuators. A, 95, 29, 2000.

5. R. F. Wolffenbutel, K. M. Mahmoud and P.P.L Regtien, Compliant capacitive wrist sensor for use in industrial robots, IEEE Trans. on Instrum. and Meas., 39:6, 991, 1990.

6. S.M. Huang et al., Electronic transducers for industrial measurement of low value capacitances, J. of Phys. E:Sci. Instrum., 21, 242, 1988.

7. R. B. McIntosh, P. E. Mauger, and S. R. Patterson, Capacitive transducers with curved electrodes, IEEE, sensors, 6:1, 125, 2006.

8. K. Kasten, J. Amelung, and W. Mokawa, CMOScompatible capacitive high temperature pressure sensors, Sens. Actuators A, 85, 147, 2000.

9. S. T. Moe et al., Capacitive differential pressure sensor for harsh environments, Sens. Actuators A, 83, 30, 2000. 
10. S. Guo, J. Guo, and W. H. Ko, A monolithically integrated surface micromachined touch mode capacitive pressure sensor, Sens. Actuators A, 80, 224, 2000.

11. H. Golnabi, Guardring effects on capacitive transducer systems. Scientia Iranica, 7, 25, 2000.

12. G. E. Fasching, W. J. Loudin, and N. S. Smith, Capacitance system for three-dimensional imaging of fluidized-bed density, IEEE Trans. On Instrumentation and Measurement, 43, 56, 1994.

13. E. D. Tsamis and J. N. Avaritsiotis, Design of a planar capacitive type senor for water content monitoring in a production line, Sens. Actuators. A, 118, 202, 2005.

14. C. N. Strizzolo and J. Cinverti, Capacitance sensors for measurement of phase volume fraction in two-phase pipelines, IEEE Trans. On Instrumentation and Measurement, 42,726, 1993.

15. H. Rodjegard, and A. Loof, A differential chargetransfer readout circuit for multiple output capacitive sensors, Sens. Actuators A, 119, 309, 2005.
16. A. Ashrafi and H. Golnabi, A high precision method for measuring very small capacitance changes. Rev. Sci. Instrum, 70, 3483, 1999.

17. S. E. Woodard and B. D. Taylor, A wireless fluidlevel measurement technique, Sens. Actuators A, 137, 268, 2007.

18. Sanwa Electric Instrument Co. Ltd. Internet Gateway at http://www.sanwa-meter.co.jp/.

19. A. L. Stott, R. G. Green, and K. Seraji, Comparison of the use of internal and external electrodes for the measurement of the capacitance and conductance of fluids in pipes. J. Phys. E: Sci. Instrum., 18, 587, 1985.

20. S. M. Huang et al., Conductivity effects on capacitance measurements of two-component fluids using the charge transfer method, J. Phys. E: Sci. Instrum., 21, 539, 1988.

21. Hach Company, Cat No. 51800-18, sension 5 Conductivity Meter Manual. 